\title{
Efficacy and effectiveness of treatment
}

$\mathrm{W}$ e as physicians usually feel rewarded when our diagnostic hypothesis is confirmed. The answer to the quiz was correct. So the next step is to define the treatment, usually involving the prescription of appropriate medication. Naturally, after that we have the feeling that a good job has been done; but this is only almost correct.

Although the diagnosis and the therapeutic are correct the most important aim is to improve the patient's health. Obviously to reach such an objective the diagnosis must be correct and the prescribed drug needs to have good efficacy, but the treatment must have effectiveness. Efficacy is treatment's defined as the treatment's effect under ideal conditions. In other words, usually the patient is in the hospital, under rigorous control and definitily receives the treatment as it should be applied.

Effectiveness is defined as the treatment's effect in the real world of medical practice. The treatment is prescribed, the patient returns to his place of origin on his own. At this point the effect of the treatment is at many risks. The effectiveness of the treatment will depend on the access he has to, for instance, the drug.

He needs to be to able to obtain it (the drug) or other type of treatment (usually requiring cash), also the patient needs to comply with the treatment. This aspect depends on many factors such as, confidence that the patient may have in the doctor who prescribed the treatment, the adverse effects of the drug, discipline, self esteem, religious belief etc.

The doctor may improve the patient's adherence or compliance, by spending some time explaining what are the expected effects of the treatment and what to do to prevent pitfalls in the treatment.

Suppose that for one specific treatment in a particular group of patients efficacy is $90 \%$, adherence of doctor is $90 \%$, and access to treatment is $80 \%$, at the end of the day, the probability of such prescriptions having been efficacious is equal to $0,9 \times 0,9 \times 0,8 \times 0,8=\underline{51.9 \%}$.

We must emphasize that the above example for chronic diseases is a very optimistic one.

For instance, more than $50 \%$ effectiveness is seldom obtained in the treatment of patients with primary hypertensions.

This means that after the correct diagnosis has been given and the most efficacious prescription for the treatment prescribed, a new challenge awaits the doctor; the treatment effectiveness. Probably that explains why the medical practice is so exciting.

In this case the intention to treat is not enough. Comprehensive evolution of patient's life as a whole and careful explanation of the why and how of the treatment may be of help. Researching how to improve patients adherance to treatment is badly needed in order to obtain more benefits from the already existent therapeutics resources.
- MD, PhD, MSc

Department of Internal Medicine, Escola Paulista de Medicina Chairman, Editor, São Paulo Medical Journal. 